\title{
CINAEDUS, NUEVO GRAFITO EN SIGILATA HISPÁNICA
}

\author{
DAVID MARTÍNEZ-CHICO \\ Universitat de València \\ david_ele@live.com \\ ORCID: 0000-0001-9907-3020
}

\section{RESUMEN}

El artículo tiene como objetivo editar y estudiar un fragmento cerámico con epigrafía incisa. El fragmento puede datarse durante el primer tercio del siglo II d.C. La palabra escrita se trata de cinaedus, que puede ser traducida como maricón o marica. Realmente se trata de un grafiti común en Pompeya y en otros soportes perdurables de escritura.

PALABRAS CLAVE: sexualidad, sociedad romana, epigrafía latina, cerámica.

\section{CINAEDUS, NEW GRAFITTI ON HISPANIC SIGILATA}

\section{ABSTRACT}

The article aims to edit and study a example of incised epigraphy on a ceramic fragment. The graffiti can be dated to the first third of the 2 nd century AD. The term refers to cinaedus, which can be translated as a fagot. It is a common graffiti in Pompeii and other abiding mediums of writing.

KEYWORDS: sexuality, Roman society, Latin epigraphy, ceramics.

Recientemenos hemos tenido acceso a un interesante testimonio epigráfico, en soporte cerámico. ${ }^{1} \mathrm{El}$ fragmento cerámico fue hallado ya hace unos años en un punto indeterminado de Burgos, seguramente en un yacimiento rural de tipo villa. El fragmento conservado tiene una anchura de 8 $\mathrm{cm}$, de extremo a extremo por largo. Se trata de una forma abierta de carena baja, morfológicamente afín a los platos/cuencos del siglo I d.C., en sigilata hispánica y con restos todavía de barniz rojo. La singularidad radica en que en la parte exterior del recipiente se grabó post coctionem un grafiti (Figs. 1 y 2), cuyo grabado se adaptó a la curvatura de la cerámica. Las letras del pequeño epígrafe poseen una altura de $16 \mathrm{~mm}$. El estilo escriturario apunta a una letra mayúscula, aunque la incisión es hasta cierto punto descuidada.

En cuanto a su lectura, pese a que la primera palabra no la tengamos completa, contamos con un primer signo conservado, en concreto el final de un trazo vertical. Pueden proponerse dos posibilidades. Pero ambas, tanto [---]ius como [---]nus, aludirían a un nombre masculino en nominativo. Mucho menos

\footnotetext{
${ }^{1}$ Queremos expresar nuestros agradecimientos a J. M. Bustillo (Alicante, Your Antiquarian Ancient Art \& Ancient Coins), ya que nos dejó estudiar su pieza. También a los evaluadores anónimos de la revista sus comentarios y sugerencias, pues permitieron mejorar la versión final del artículo.
} 
problemática es la segunda palabra, perfectamente leída como cinaedus, bien documentada en Pompeya, ${ }^{2}$ y donde es especialmente conocida. ${ }^{3}$ Sin embargo, en nuestro texto nos encontramos ante cinedus, con una $\mathrm{E}$ de dos barras verticales. La única forma que podría interpretarse como cursiva es esta última E, pero no es una forma determinante, puesto que aparece también en la capital. En consecuencia, no puede decirse que la inscripción esté en cursiva. La monoptongación del diptongo ae $>$ e es un fenómeno muy frecuente. Además, la fórmula nomen + cinedus/cinaedus está también documentada: cítese Lasius cinedus, ${ }^{4}$ es decir, 'Lasio, es marica', o Iustus cinaedu[---], también "marica".

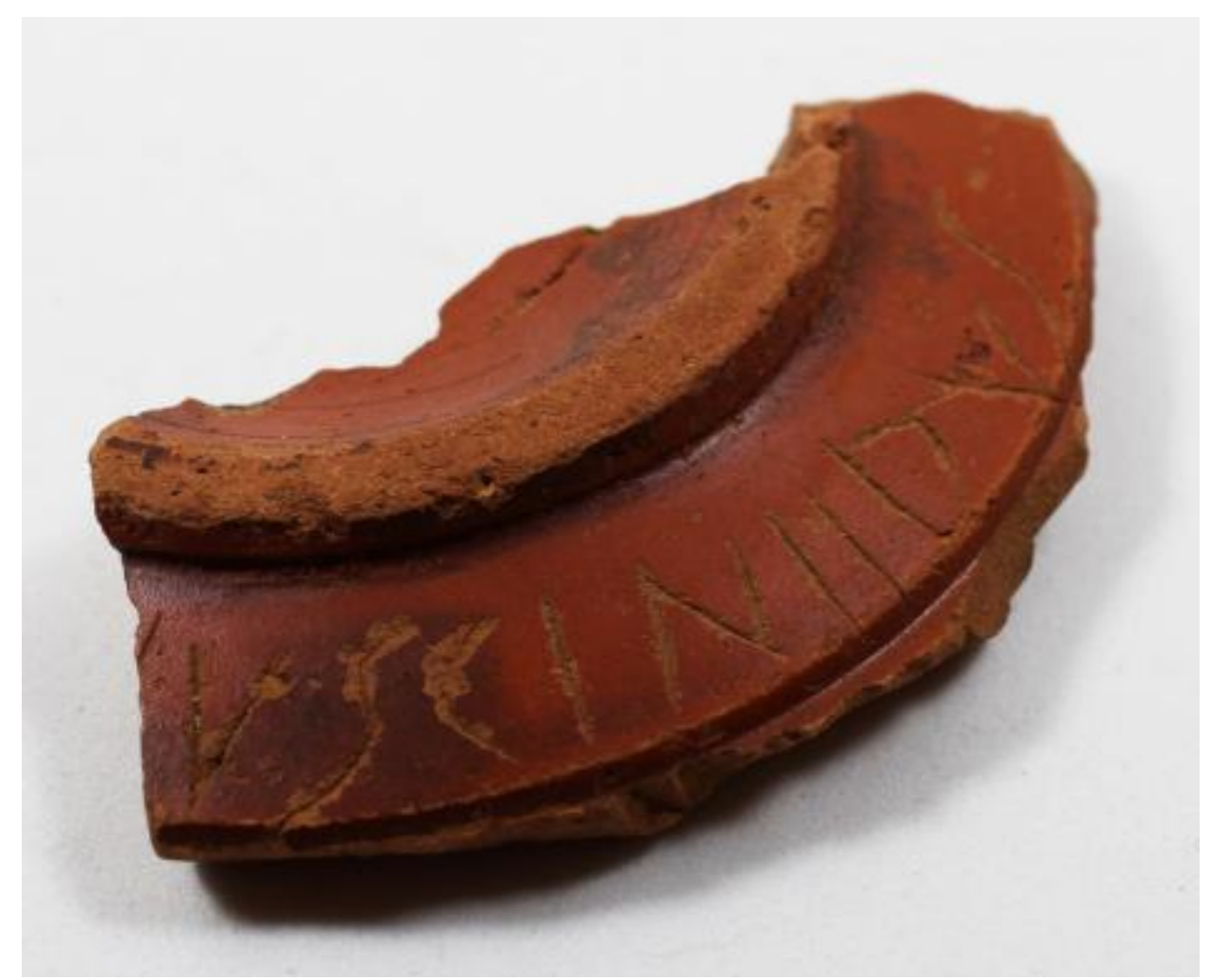

Figura 1. Imagen de la sigilata hispánica con el grafiti.

${ }^{2}$ A modo de ejemplos, pueden citarse CIL IV, 1485a, 1825, 2332, 3079, 3336 o 4917.

${ }^{3}$ Levin-Richardson 2019: 40-63.

${ }^{4}$ CIL IV $10671=$ EDR 154575.

${ }^{5}$ CIL IV $10654 \mathrm{c}=E D R 154572$. 


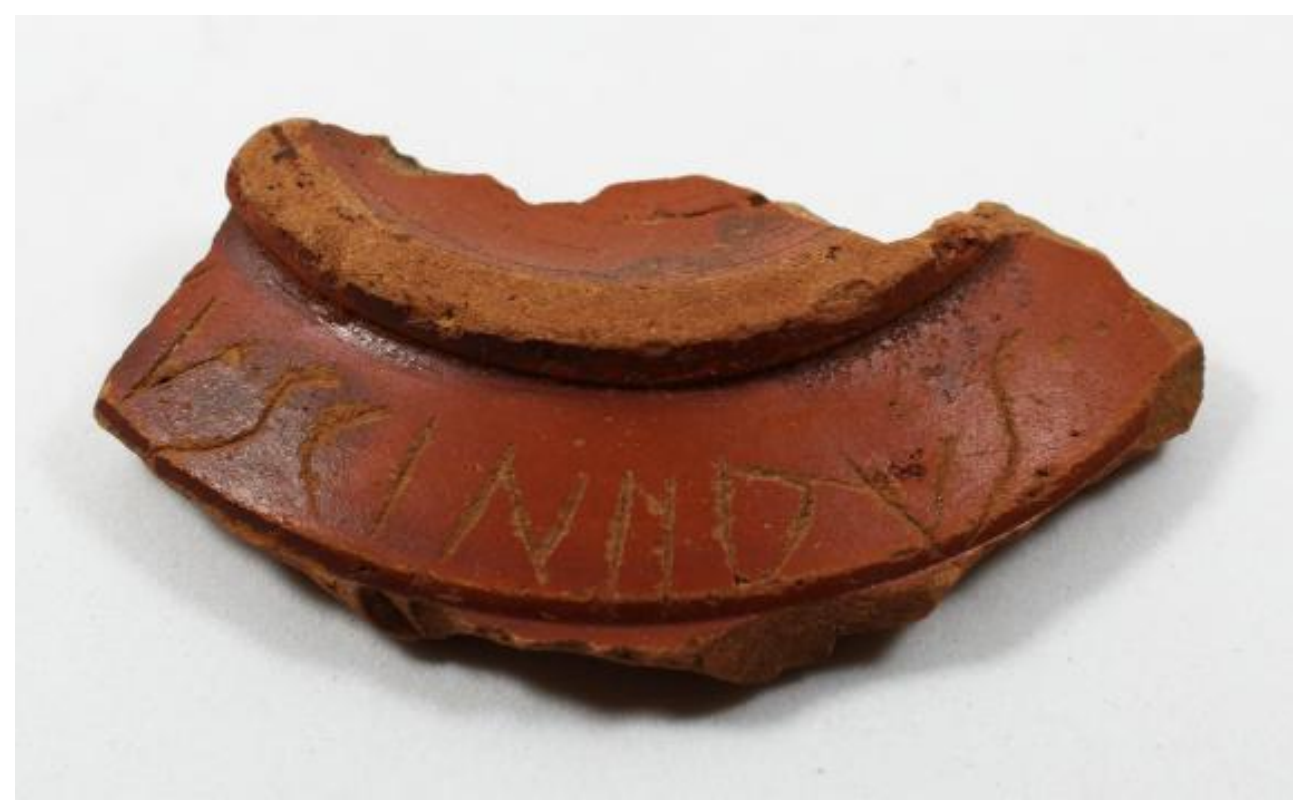

Figura 2. Imagen dispuesta horizontalmente respecto al grafiti.

Cinaedus es un término latino de índole sexual y despectiva. Hoy día esta palabra se suele traducir como marica, maricón y/o sodomita. Ciertamente cinaedus es un catamito y, por lo tanto, es siempre masculino. Cinaedus y cinaedia derivan del griego kívaıঠos, que entre los helenos designaba tanto al pescado como al sodomita. A raíz de este término y su problemático significado, Kamen y Levin-Richardson, afirman que no hay adjetivo actual para describir al cinaedus, pues los romanos lo expresaban en referencia al deseo de ser penetrado analmente y su movimiento corporal durante este acto. ${ }^{6}$ Williams, uno de los mayores expertos en masculinidad romana, afirmó que un cinaedus no era un "marica", un "hada" o una "reina", pero que a falta de una palabra mejor en nuestro vocabulario occidental, cualquier traductor tiene que tomar una decisión consciente, al margen de los prejuicios occidentales, oprimidos por una 'heterosexualidad obligatoria'. ${ }^{7}$ Nosotros optamos por un criterio conservador.

En otros ejemplares hispanos, el propietario del recipiente cerámico se dirige al posible lector o ladrón, que se le tacha de cinaedus, como bien demuestra el ejemplar hallado en Santa Criz de Eslava, con VAS[---] / cin(edus) / Acut[ius]; la secuencia el vas- puede referirse a vasus, si bien en latín esta forma es rara frente a la común vas, vasis. ${ }^{8}$ El repertorio de grafitis hispanos sobre cerámicas es muy heterogéneo, ${ }^{9}$ aunque el elenco referido a sexualidad todavía es algo escaso. El último publicado, también sobre sigilata hispánica, reproduce el sustantivo femenino fellatrix. ${ }^{10}$

\footnotetext{
${ }^{6}$ Kamen y Levin-Richardson 2015: 453-455.

${ }^{7}$ Williams 1999: 217.

${ }^{8}$ Andreu Pintado et al. 2019: 96.

${ }^{9}$ Étienne et al. 1976: 144-205, no 306-443b; Polo López et al. 1999.

${ }^{10}$ Rodríguez Morales y Gómez-Pantoja 2020.
} 
Aunque no es el caso, resulta también interesante la transformación de la vocal breve I en E -de cinaedus a cenaedus-, corriente en el latín vulgar y en grafitis muy raros. Es el caso estudiado por Andreu Pintado y Delage González, que interpretan su grafiti, procedente de Los Bañales, como qui legerit cen[aedus], es decir, 'maricón, el que lo lea'; sin obviar otras posibles secuencias, como qui legerit cen[am], 'el que lo lea, que compre la cena'. ${ }^{11}$ En realidad el vulgarismo cenaedus por cinaedus es muy raro y se restringe al ejemplar de Los Bañales.

BIBLIOGRAFÍA

Andreu Pintado, J., OzCÁRIZ GIL, P. y MATEO PÉREZ, T. (2019), Epigrafía romana de Santa Criz de Eslava (Eslava, Navarra), Faenza.

ANDREU PiNTADO, J. y DELAGE GONZÁLEZ, I. (2017), “Un singular grafito sobre sigilata hispánica hallado en Los Bañales de Uncastillo (Zaragoza)", Ficheiro Epigráfico 152, inscripción 609.

CIL IV = ZANGEMEISTER, C. y SCHOENe, R. (1871), Corpus Inscriptionum Latinarum. Vol IV: Inscriptiones Parietariae Pompeianae Herculanenses Stabianae, Berlín.

$E D R=A A . V V .$, "Epigraphic Database Roma", en www.edr-edr.it/default/index.php (consultada el 15/05/2020).

Étienne, R., FAbre, G., Leveque P. y Leveque, M. (1976), Fouilles de Conimbriga, II. Épigraphie et sculpture, París.

KAMEN D. y LEVIN-RICHARDSON, S. (2015), “Revisiting Roman Sexuality: Agency and the Conceptualization of Penetrated Males", en M. Masterson, N. S. Rabinowitz y J. Robson (eds.), Sex in Antiquity: Exploring Gender and Sexuality in the Ancient World, Routledge, Oxon-New York, pp. 449-460.

LEVIN-RICHARDSON, S. (2019), The Brothel of Pompeii. Sex, Class, and Gender at the Margins of Roman Society, Cambridge-New York.

Polo LóPEZ, J., SÁNCHEZ-LAFUENTE PÉREZ, J. y RASCón MARQuÉS, S., (1999), “Grafitos sobre instrumental doméstico en Hispania", en XI Congreso Internazionale di Epigrafia Greca e Latina (Roma 1997), Roma 1999, pp. 583-599.

Rodríguez Morales, J. y GÓmEZ-PANTOJA, J. L. (2020), “Fellatrix”, Ficheiro Epigráfico 198, inscripción 728.

WiLliams, C. A. (1999), Roman Homosexuality: Ideologies of Masculinity in Classical Antiquity, New York-Oxford.

${ }^{11}$ Andreu Pintado y Delage González 2017. 